\title{
Genetic Variation and Structure in Remnant Population of Critically Endangered Melicope zahlbruckneri
}

\section{Raji $\mathrm{JA}^{1,2^{*}}$ and Atkinson $\mathrm{CT}^{3}$}

${ }^{1}$ Hawai'i Cooperative Studies Unit, University of Hawaii at Hilo, HI, USA

${ }^{2}$ U.S. Geological Survey, Pacific Island Ecosystems Research Center, HI, USA

${ }^{3}$ Agronomy Department, lowa State University, Ames, IA, USA

"Corresponding author: Raji, JA, Hawai'i Cooperative Studies Unit, University of Hawaii at Hilo, 200 W. Kawili St. Hilo, HI, USA, Tel: +8089330759; E-mail: j.raji@outlook.com; jbr80@iastate.edu

Received date: November 16, 2016; Accepted date: December 13, 2016; Published date: December 19, 2016

Copyright: @ 2016 Raji JA, et al. This is an open-access article distributed under the terms of the Creative Commons Attribution License, which permits unrestricted use, distribution and reproduction in any medium, provided the original author and source are credited.

\begin{abstract}
The distribution and amount of genetic variation within and between populations of plant species are important for their adaptability to future habitat changes and also critical for their restoration and overall management. This study was initiated to assess the genetic status of the remnant population of Melicope zahlbruckneri-a critically endangered species in Hawaii, and to determine the extent of its genetic variation and diversity in order to propose rational conservation approaches. Molecular marker allele frequencies identified genetic groups with low overall differentiation but identified the most genetically diverse individuals within the population. Analysis of Amplified Fragment Length Polymorphic (AFLP) marker loci in the population based on Bayesian model and multivariate statistics classified the population into four subgroups. We inferred a mixed species population structure based on Bayesian clustering and frequency of unique alleles. The percentage of Polymorphic Fragment (PPF) ranged from 18.8 to $64.6 \%$ for all marker loci with an average of $54.9 \%$ within the population. Inclusion of all surviving $M$. zahlbruckneri trees in future restorative planting at new sites are suggested, and approaches for longer term maintenance of genetic variability are discussed. To our knowledge, this study represents the first report of molecular genetic analysis of the remaining population of $M$. zahlbruckneri and also illustrates the importance of genetic variability for conservation of a small endangered population.
\end{abstract}

Keywords: Endangered species; Genetic conservation; Melicope zahlbruckneri; Molecular markers

\section{Introduction}

Rare and endangered species are generally vulnerable to environmental changes and genetic risks. Due to their often fragmented and small population sizes [1]. They are more likely to have limited fitness along with a fixation of recessive and deleterious mutations. This increases the risk of genetic variability loss through random genetic drift when compared with larger and more diverse populations [2]. Preservation of natural genetic diversity levels and structure is thus critical for the long term management of these endangered and threatened species to prevent further loss of diversity that could arise from unexpected fluctuations in their habitat and the environment [3].

Melicope zahlbruckneri is endemic to the Island of Hawaii with a small and declining population. It belongs to the Rutaceae family and listed as federally endangered. This species currently occurs as a single population at the Hawaii Volcanoes National Park with less than 30 individuals left in the wild. While there is little published information on the reproductive ecology of $M$. zahlbruckneri, it is believed to be outcrossing, with pollination and seed dispersal by birds. Species in this family generally have flowers that divide into four or five parts. In terms of size, individual plants range from herbs to shrubs and small trees. M. zahlbruckneri grows up to about $10-12 \mathrm{~m}$ tall with mature leaves that are about $6-24 \mathrm{~cm}$ long and $4-12.5 \mathrm{~cm}$ wide, with welldefined lateral veins [4]. There is currently no information available on the genetics of the species, also, very little is known on their historic distribution. Thus, it is important to determine the current level of genetic variability and structure in the remnant population to aid development of sampling strategies for population restoration and propose informed recommendations to assist their ongoing management.

Molecular markers have proved to be efficient means of investigating population structure, genetic diversity, and are important components in genetic conservation studies of different species $[5,6]$. Molecular markers are able to provide a precise understanding of genetic diversity through the identification of genomic segments that differentiate individuals or population without the need for genetic information about the genome $[7,8]$. Neutral markers in combination with geographic information and appropriate statistical tools have been used frequently for the study of genetic diversity in a wide range of plant species [9-12] and have been especially useful for studies involving intra and inter-species genotypic variation in both large and small populations [13].

Several molecular markers are available with the choice often determined by availability of information for the species, the population type and size, and available resources. Microsatellites and Single Nucleotide Polymorphic (SNP) markers are recognized as the most efficient at revealing genetic diversity within and between species and can distinguish homozygous and heterozygous individuals when pedigree information is desired. However, the use of these codominant markers requires the availability of genomic sequence information about the species as well as high cost consideration for the 
initial marker development particularly in studies involving natural populations where several species families are involved. Amplified Fragment Length Polymorphism (AFLP) on the other hand has the potential to screen a large number of genetic loci and does not require prior information about the genome of the species under investigation. Here we report an AFLP-based molecular assessment of $M$. zahlbruckneri to evaluate the genetic structure of the remnant population actively managed at the Hawai'i Volcanoes National Park.

\section{Materials and Methods}

\section{Sample collection and DNA isolation}

The M. zahlbruckneri population was sampled by collecting leaf tissue from all known and accessible individual trees of the species.

For each individual, a small sized healthy leaf was chosen and stored on ice in a sealed plastic bag. Leaf samples were taken to the laboratory for immediate processing or stored at $-70^{\circ} \mathrm{C}$ until DNA extraction. A total of 28 samples were collected from the Kipukapuaulu (Table 1), the only remaining site where the species is known to be extant [except for a previously reported individual tree back in 1997 at Laupahoehoe [14].

DNA was extracted using a modified plant DNA isolation protocol by Dellaporta et al. with minor modifications [15]. The concentration and purity of DNA was measured using the nanodrop ND-1000 spectrophotometer and quality was assessed on agarose gel.

\section{SSR marker transferability test}

Simple Sequence Repeats (SSRs) have had limited use in studies involving native plant populations in Hawaii because they have to be developed specifically for each plant species. However, because several studies have demonstrated the successful transferability of gene based microsatellites across related genera in crop plants [11,16,17], we decided to test available genomic or gene based microsatellites that have been developed for other taxa close to M. zahlbruckneri to explore the prospects of transferability of such markers to genotype $M$. zahlbruckneri population.

Primer sequences of seven compound microsatellite loci developed for Melicope quadrilocularis [18] were synthesized (Integrated DNA Technologies, Inc. Corvallis, IA) for testing. We optimized PCR reaction components and cycling parameters for each primer pair and tested them for successful amplification and polymorphism detection in Melicope zahlbruckneri population. PCR reactions for SSR marker assay were performed in a Biorad DNA Engine (Peltier Thermal Cycler); PCR products were first separated on $1.5 \%$ TAE agarose gels and visualized under UV after ethidium bromide staining and then analyzed by capillary electrophoresis using an automated Genetic Analyzer ABI 3730.

\section{AFLP restriction digestion and amplification}

The AFLP procedure followed the original method described by Vos et al. with a few modifications, and unless otherwise indicated, Life Technologies (Invitrogen and Applied Biosystems) reagents were used in all reaction procedures [19]. Total genomic DNA (500 ng) was digested with 4.0 units of EcoRI and MseI restriction enzymes at $37^{\circ} \mathrm{C}$ for $4 \mathrm{~h}$ in a $25 \mu \mathrm{l}$ reaction volume that included $2.5 \mu \mathrm{l}$ of $5 \mathrm{X}$ reaction buffer and $0.2 \mu \mathrm{l}$ of $10 \mathrm{mg} / \mathrm{ml}$ bovine serum albumin (BSA). After digestion, a $5 \mu$ aliquot of digested DNA from each sample was run on
TAE 1\% agarose gel to check for complete and uniform digestion. Ligation reaction was performed in a $40 \mu$ solution and contained 20 $\mu \mathrm{l}$ of the digested DNA, 5 pmol of the EcoRI adapter, 50 pmol of the MseI adapter, 1 units T4 DNA ligase, $0.10 \mu \mathrm{l}$ of $10 \mathrm{mg} / \mathrm{ml} \mathrm{BSA}(1 \mu \mathrm{g})$, and $4 \mu \mathrm{L}$ DNA Ligase buffer. The reaction was left to incubate for $3 \mathrm{~h}$ at $20^{\circ} \mathrm{C}$.

Pre amplification reaction was performed in a $30 \mu$ volume using 1 $\mu \mathrm{l}$ of the ligation reaction as the template, and combined with $21.5 \mathrm{ng}$ each of the EcoRI+A and MseI+A primers, 3.0 $\mu$ Amplitaq 360 DNA buffer ( $500 \mathrm{mMKCl}$ ), $15 \mathrm{mM} \mathrm{MgCl}_{2}, 10 \mathrm{mM}$ dNTPs (each at $2.5 \mathrm{mM}$ ), and 1 unit of Taq DNA polymerase. The reaction was processed in a thermal cycler using the following cycling parameters: an initial denaturation step at $94^{\circ} \mathrm{C}$ for $60 \mathrm{~s}, 20$ cycles of $94^{\circ} \mathrm{C} / 30 \mathrm{~s}, 56^{\circ} \mathrm{C} / 60 \mathrm{~s}$, $72^{\circ} \mathrm{C} / 60 \mathrm{~s}$ and a final extension at $72^{\circ} \mathrm{C}$ for $10 \mathrm{~min}$. Selective amplification was performed using 36 EcoRI/MseI primer combinations to genotype the population. The primer pairs were chosen based on the number, clarity, and reproducibility of fragments produced in a preliminary screening of 60 primer combinations. Selective amplification was performed in a $10 \mu \mathrm{l}$ reaction volume containing $2.5 \mu \mathrm{l}$ of diluted pre amplification reaction (diluted 1:20 in $1 \times$ Tris-ethylenediaminetetraacetic acid buffer), $5 \mathrm{ng}$ of EcoRI +3 and $15 \mathrm{ng}$ of MseI+3 selective primers, $1 \mu \mathrm{l}$ Amplitaq 360 buffer, $0.24 \mu \mathrm{l}$ dNTPs $(10 \mathrm{mM})$, and 0.2 units of Amplitaq 360 DNA polymerase. All EcoRI+3 selective primers were fluorescently labeled with FAM, NED and JOE using the DS-30 Matrix standard (Applied Biosystems, Foster City, CA). Amplification was performed with a touch down cycling procedure as follows: An initial denaturation step at $94^{\circ} \mathrm{C}$ for $2 \mathrm{~min}, 1$ cycle of $30 \mathrm{~s}$ at $94^{\circ} \mathrm{C}, 30 \mathrm{~s}$ at $65^{\circ} \mathrm{C}$, and $60 \mathrm{~s}$ at $72^{\circ} \mathrm{C}$, followed by 11 cycles in which the annealing temperature decreases $0.7^{\circ} \mathrm{C}$ per cycle, and 22 cycles of $30 \mathrm{~s}$ at $94^{\circ} \mathrm{C}, 30 \mathrm{~s}$ at $56^{\circ} \mathrm{C}$ and $60 \mathrm{~s}$ at $72^{\circ} \mathrm{C}$, and a final extension step at $72^{\circ} \mathrm{C}$ for $5 \mathrm{~min}$.

\section{Sample genotyping and pre-analysis of data}

AFLP fragment analysis of all individuals was performed by capillary electrophoresis using automated Genetic Analyzer ABI 3730. ROX labeled size standard that range in size from 35-500 bp was used for fragment sizing. AFLP fragment data were normalized based on local southern size-call algorithm, peak saturation, baseline saturation, pull-up correction, and spike removal corrections. Following normalization, allele sizing and call procedures were performed using the GENEMAPPER (ver. 4.1, Applied Biosystems) software program. Individual peaks were called on the basis of the total signal intensity and a peak was scored only if its intensity exceeded a fixed threshold. Data from a standardized template was used for each primer combination to ensure that peak sizing and position was precise and reproducible in all samples.

\section{Data analysis}

Marker fragments were scored as either present or absent to create a binary matrix for each individual. The resulting binary matrix was used as input data to generate Jaccard's similarity coefficient and then transformed into a dissimilarity measure as (1-Jaccard's similarity) using the SIMQUAL module of NTSYSpcver. 2.20d program [20-21]. Cluster analysis was performed using the unweight pair group method with arithmetic averages (UPGMA). The resulting tree branching pattern was evaluated by bootstrapping based on 1000 replicates (PHYLIP software package v.3.66) [22]. Genetic variation was further assessed by using the TREECON program to generate Neighbor joining cladograms. Additionally, genetic diversity and allele frequency 
Citation: Raji JA, Atkinson CT (2016) Genetic Variation and Structure in Remnant Population of Critically Endangered Melicope zahlbruckneri. J

Page 3 of 8

measures for the population were calculated using the AFLP-SURV program [23]. Hardy-Weinberg equilibrium (FIS=0) was assumed for the estimation of allele frequencies as an essential requirement for dominant markers where the absent fragment frequency is q2 (q being the null allele frequency). Furthermore, Nei's unbiased expected heterozygosis was used to calculate genetic diversity within the population as the percentage of within-population polymorphic loci relative to overall polymorphic loci. This is based on the assumption that the presence of a fragment indicates either a heterozygote or homozygote for the dominant allele with frequencies estimated based on the null allele frequency. To further examine the genetic structure of M. zahlbruckneri population, a model based Bayesian analysis was performed using the program STRUCTURE ver. 2.4 [24-26]. A nonuniform prior distribution of allelic frequencies was assumed with its parameters derived from the observed distribution of fragment frequencies among AFLP loci [27]. Log likelihood and estimates for allele frequency divergence as a result of random drift (FST) were computed using the admixture model and correlated allele frequencies options with a burn-in period of 80,000 and 100,000 Markov Chain Monte Carlo (MCMC) iterations after burn in. Likely genetic clusters (K) groups were set over a range from $\mathrm{K}=1-5$ with 10 replicate runs at each $\mathrm{K}$. The posterior probability $\mathrm{P}(\mathrm{X} \mid \mathrm{K})$ was estimated to give an indication of the true likely number of groups without prior information about the population. Results from STRUCTURE were further analyzed and collated with the structure harvester program
[28]. The CLUMPP program was used for permutations of the most likely observations among replicate runs for each $\mathrm{K}$, and results were visualized in the DISTRUCT program $[29,30]$

Principal Coordinate Analysis (Pcoa) was used to explore genetic relatedness of individuals based on the modified Roger's distance matrices generated from the binary data populations. PCoA computations were performed with the MVSP (Ver.3.21) program. A plot of the population was produced to show distances among samples in the plot as likely illustration of genetic distances within the population. The Mantel statistics was used to assess correlation between observed spatial and genetic distances among individuals.

\section{Results}

\section{Microsatellites marker analysis}

Twenty-eight plant samples from the M. zahlbruckneri population were genotyped using the seven compound microsatellite primers developed for Melicope quadrilocularis [18]. Five of the seven SSR's amplified expected allele sizes for each primer pair as described for $M$. quadrilocularis. Although transferability of the SSRs was confirmed in M. zahlbrukneri, the markers were unable to detect polymorphism in M. zahlbruckneri samples. Consequently, we decided to focus the rest of the study on the AFLP marker analysis results.

\begin{tabular}{|c|c|c|c|c|}
\hline $\begin{array}{l}\text { Selective Sequence } \\
\text { E/M Pairs }\end{array}$ & $\begin{array}{l}\text { Total No } \\
\text { of Loci }\end{array}$ & $\begin{array}{l}\text { Polymorphic } \\
\text { Loci }\end{array}$ & $\begin{array}{l}\text { Polymorphic } \\
\text { Fragments (\%) }\end{array}$ & $\begin{array}{l}\text { Size Range } \\
\text { (Base pair) }\end{array}$ \\
\hline E-AAC/M-CTC & 130 & 84 & 64.6 & $50-480$ \\
\hline E-ACT/M-CTT & 189 & 114 & 60.3 & $50-500$ \\
\hline E-AAG/M-CAC & 145 & 85 & 58.6 & $45-450$ \\
\hline E-ACA/M-CAC & 172 & 96 & 55.8 & $50-500$ \\
\hline E-ACC/M-CAC & 136 & 75 & 55.1 & $65-480$ \\
\hline E-ACC/M-CAT & 130 & 80 & 61.5 & $55-500$ \\
\hline E-AGC/M-CTA & 142 & 81 & 57.0 & $50-480$ \\
\hline E-AGC/M-CAC & 106 & 66 & 62.3 & $50-500$ \\
\hline E-AGG/M-CTA & 132 & 80 & 60.6 & $60-500$ \\
\hline E-AGG/M-CAG & 135 & 73 & 54.0 & $50-500$ \\
\hline E-ACT/M-CAG & 134 & 72 & 53.7 & $50-490$ \\
\hline E-ACG/M-CTC & 167 & 95 & 56.8 & $50-475$ \\
\hline E-ACA/M-CTG & 155 & 87 & 56.1 & $55-480$ \\
\hline E-AAC/M-CTT & 102 & 59 & 57.8 & $50-500$ \\
\hline E-ACG/M-CTT & 103 & 39 & 37.9 & $40-500$ \\
\hline Е-АСТ/M-СТC & 150 & 85 & 56.6 & $50-500$ \\
\hline E-AAG/M-CTC & 137 & 78 & 56.9 & $45-500$ \\
\hline E-AAC/M-CAG & 140 & 67 & 47.9 & $40-500$ \\
\hline E-АCT/M-СTG & 185 & 110 & 59.4 & $50-500$ \\
\hline
\end{tabular}


Citation: Raji JA, Atkinson CT (2016) Genetic Variation and Structure in Remnant Population of Critically Endangered Melicope zahlbruckneri. J

\begin{tabular}{|c|c|c|c|c|}
\hline E-ACA/M-CTC & 157 & 92 & 58.5 & $60-500$ \\
\hline E-ACC/M-CAG & 127 & 65 & 51.8 & $64-500$ \\
\hline E-AGG/M-CTT & 101 & 40 & 39.6 & $50-500$ \\
\hline E-ACC/M-CTG & 100 & 62 & 62.0 & $45-490$ \\
\hline E-AAG/M-CTT & 85 & 16 & 18.8 & $40-500$ \\
\hline E-AGG/M-CTG & 97 & 61 & 62.9 & $55-480$ \\
\hline E-ACA/M-CTT & 96 & 25 & 26.0 & $50-500$ \\
\hline E-AAC/M-CAT & 145 & 81 & 55.0 & $40-500$ \\
\hline E-AAC/M-CTA & 135 & 77 & 57.0 & $40-500$ \\
\hline E-ACT/M-CAT & 168 & 101 & 60.2 & $40-490$ \\
\hline E-ACA/M-CTA & 167 & 102 & 61.0 & $50-500$ \\
\hline E-ACA/M-CAT & 150 & 92 & 61.3 & $50-500$ \\
\hline E-AAC/M-CAC & 120 & 64 & 53.3 & $50-500$ \\
\hline E-АCT/M-СТА & 165 & 102 & 61.8 & $55-500$ \\
\hline E-ACG/M-CAT & 156 & 93 & 59.6 & $50-500$ \\
\hline E-ACG/M-CAG & 163 & 100 & 61.3 & $55-500$ \\
\hline E-AAG/M-CTA & 145 & 81 & 55.9 & $55-500$ \\
\hline Mean & 137.97 & 77.2 & 54.9 & - \\
\hline
\end{tabular}

Table 1: AFLP marker pairs, characteristics and average variation in selective amplification of M. zahlbruckneri.

\section{Genetic distance and population structure}

Thirty-six AFLP EcoRI/MseI combination primer pairs generated 2780 fragments (Table 2).

The percentage of polymorphic fragments per AFLP locus ranged from $18.8 \%$ to $64.6 \%$ with an average of $54.9 \%$ (Table 2 ).

Of the total fragments scored, 620 unique fragments (22.3\%) were detected in a few samples within the population. Coefficients of similarity for all possible pair-wise comparisons ranged from $0.621-1.171$.

Hierarchical trees with bootstrap values revealed low to moderate sub structuring in the M. zahlbruckneri population. Main clusters that are subdivided into smaller groups are shown as well as 2 individual entries located on separate branches away from the rest of the groups (Figure 1). Neighbour joining tree derived from Jaccard's coefficient appeared to be less differentiated than expected considering the percentage of unique alleles detected in a small population. Many of the samples that accounted for the private alleles found in the $M$. zahlbruckneri population showed genetic profiles that varied substantially from the rest of the population. MZ23 and MZ27 in particular were noticeably the most diverged from the rest of the population (Figure 2). Cluster analysis of the population was reconstructed without MZ23 and MZ27 to examine possible outlier effect but no significant effect was detected in the overall topology of the trees.
Table 2: Average distances between individuals and estimated mean value of Fst for each cluster of $M$. zahlbruckneri.

\begin{tabular}{|l|l|l|}
\hline Cluster & Average distance* & Mean Fst value \\
\hline 1 & 0.3762 & $0.0117(\mathrm{p}>0.05)$ \\
\hline 2 & 0.3661 & $0.0150(\mathrm{p}>0.05)$ \\
\hline 3 & 0.2271 & $0.3471(\mathrm{p}<0.01)$ \\
\hline 4 & 0.2317 & $0.9157(\mathrm{p}>0.01)$ \\
\hline Parameter & Value (clusters 1-4) \\
\hline Estimated Ln Prob of Data (Ln P(D)) & -33461.6 \\
\hline Mean In likelihood & -31309.1 \\
\hline \begin{tabular}{l} 
Variance of In likelihood \\
\hline Mean value of alpha
\end{tabular} & 4305 \\
\hline $\begin{array}{l}\text { *Average distance (between individuals within a cluster) } \\
\text { Ln Likelihood: log likelihood of the data given values of } P \text { (estimated allele } \\
\text { frequencies) and } Q \text { (the estimated membership coefficient for each individual in } \\
\text { a cluster). }\end{array}$ & 0.1453 \\
\hline $\begin{array}{l}\text { Est. Ln } P \text { (D): current estimate of In (PX|K)), averaging over all iterations after } \\
\text { burn in period. }\end{array}$ \\
\hline
\end{tabular}


Citation: Raji JA, Atkinson CT (2016) Genetic Variation and Structure in Remnant Population of Critically Endangered Melicope zahlbruckneri. J

Page 5 of 8

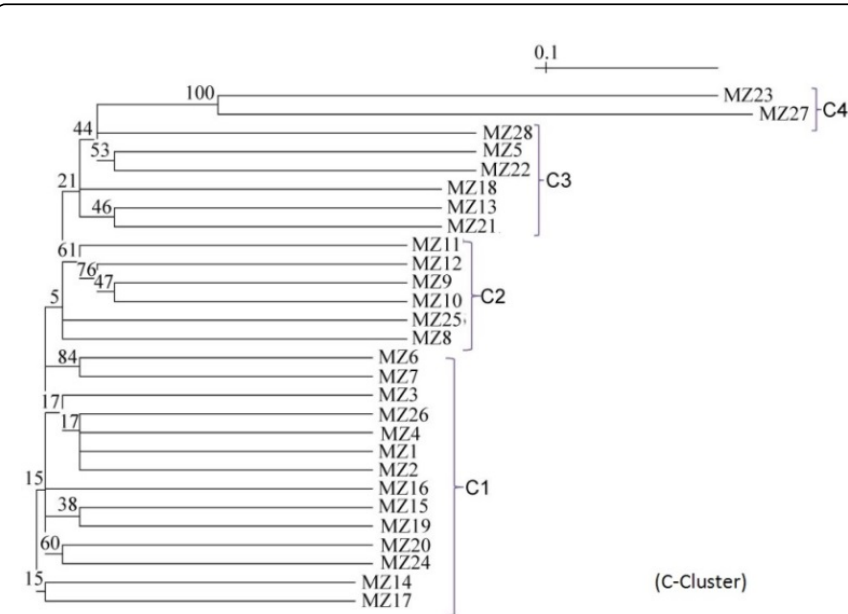

Figure 1: Hierarchical cluster analysis of M. zahlbruckneri using UPGMA (unweighted pair group method with arithmetic mean). Bootstrap values (based on 1000 permutations) are indicated at each tree node.

Bayesian analysis using the STRUCTURE program partitioned the observed population genetic variation into four clusters $(\mathrm{K})$ that are identical to those obtained from PCoA and unrooted NJ trees (Figures 2 and 3$)$.

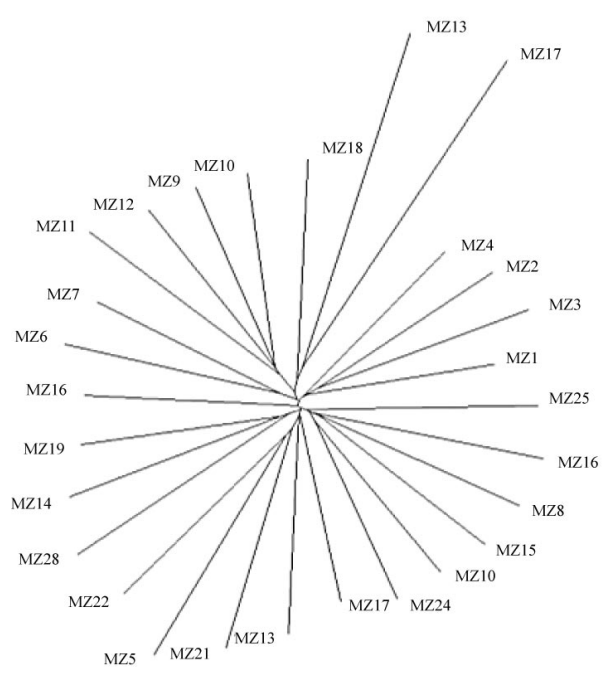

Figure 2: Unrooted neighbour joining tree illustrating genetic relationship of $28 \mathrm{M}$. zahlbruckneri individuals based on genetic similarities derived from AFLP marker.

A correlated allele frequency with admixture model was indicated for the analysis. The optimal convergence of the MCMC algorithm was achieved by using a burn-in period of 80,000 steps, followed by 100,000 steps of data collection and 10 replicated runs to ensure efficiency in clustering computation and accurate estimates of the posterior distribution. The optimal sample resolution in each cluster was obtained at $\mathrm{K}=4$. The graphical output showing the distribution and proportion of shared AFLP genomic segments is presented in
Figure 4. The plot showed the assignment of individuals into different cluster groups denoted by vertical bars with different colours.

Two individuals (MZ23 and MZ27) were significantly diverged from the rest of the population. Similarly, eight individuals (MZ3, MZ13, MZ14, MZ16, MZ24, MZ25, MZ26 and MZ28) had significant numbers of rare alleles but also shared a significant number of alleles with the rest of the population. These 8 individuals are suspected to have mixed ancestry based on the combination of unique allelic profiles leaf characteristics that seemed unusual in shape and outside of the expected size range for M. zahlbruckneri (McDaniels S., Pers. Comm). They also showed substantial degree of genetic similarities to both MZ23 and M27.

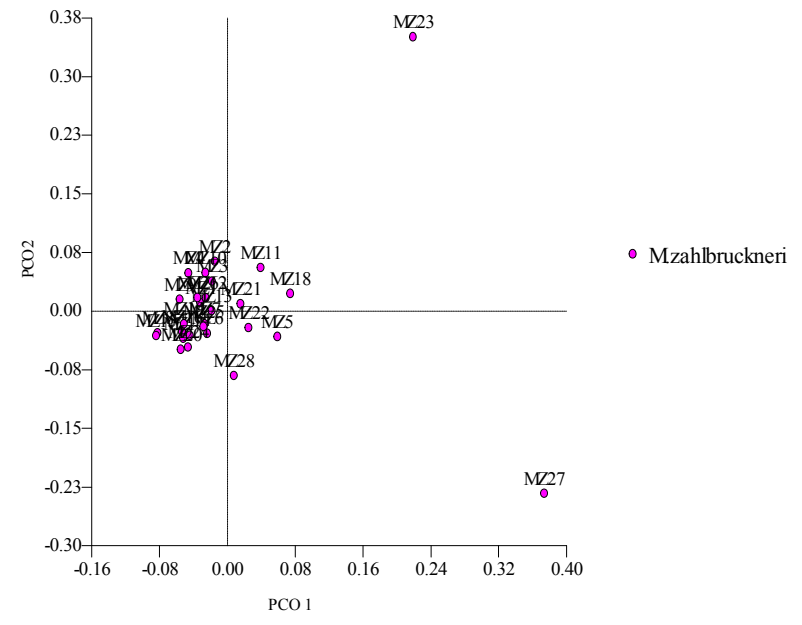

Figure 3: Principal coordinate analysis of $M$. zahlbruckneri population showing estimated genetic relationship among individuals based on AFLP loci.

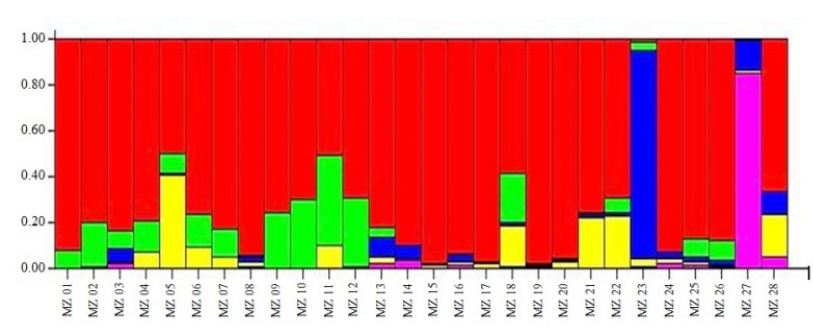

Figure 4: STRUCTURE analysis of $28 \mathrm{M}$. zahlbruckneri individuals with $\mathrm{K}=4$ clusters (blue, green, yellow, purple). The proportions of membership into the various clusters are shown on the $y$-axis shows. Individuals are represented by colored vertical bars.

The FST values of allele distribution provided additional information on allele frequency divergence estimate from possible ancestral frequencies as a result of random drift for each cluster groups. The FST values for the rarest alleles represented in the M. zahlbruckneri population ranged from 0.87-0.96 and were restricted to only a few individuals within the population. The FST values also showed the highest distance between clusters in the group comprising MZ23 and MZ27 (FST 0.92, P<0.01) and cluster 1 the lowest at FST 
$0.012, \mathrm{P}>0.05$. The average distance between individuals within each clusters varied among the 4 clusters. The highest distance score was found within individuals of cluster $1(0.3762)$ and the lowest with the members of cluster $3(0.2271)$. The mean value of log likelihood for each cluster $(\mathrm{K})$ is -31309 , the mean value of alpha is 0.1453 and the estimated value of Ln probability of data is -33461 .

\section{Genetic relatedness and population variation}

Principal coordinates analysis scores from the first three coordinates in the $M$. zahlbruckneri population showed that eigenvectors accounted for $65.82 \%$ cumulative variation of the total variance observed, with the first coordinate accounting for the most variation at $29.9 \%$. The plot of genetic distances estimated from the coordinates showed genetic grouping and relationships between individual samples that is reflective of a high degree of common alleles in more than $60 \%$ of the individuals. It also highlights the effect of unique alleles on grouping along the PCO2 axis (Figure 4).

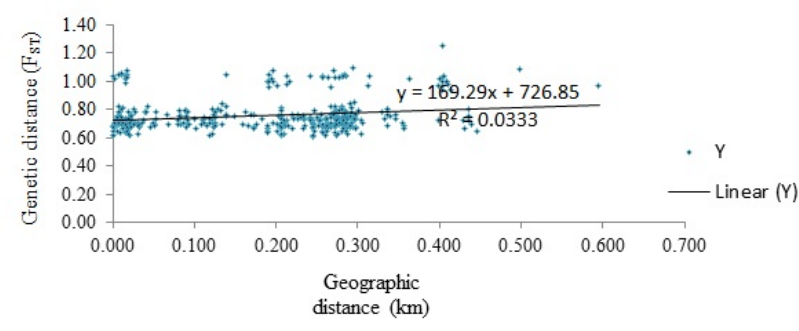

Figure 5: Mantel correlation of M. zahlbruckneri population computed from matrices of within population pairwise genetic differentiation (Nei and Li (1979)) and the natural logarithm of pairwise geographic distances.

Two individuals, MZ 23 and MZ 27 varied significantly and formed a cluster away from the rest of the population. This result is consistent with the pattern of variation observed from Bayesian analysis, NJ cladogram and UPGMA trees constructed for the population. In addition, Mantel statistics with 1000 random permutations did not indicate a significant evidence of correlation between genetic differentiation (FST) and geographical distances among individuals within the location ( $\mathrm{R} 2=0.0333, \mathrm{P}=0.708)$ (Figure 5).

\section{Discussion}

The number of polymorphic AFLP loci analyzed in this study provided reliable estimates of the overall genetic diversity, and a reasonable representation of the $M$. zahlbruckneri genetic structure despite the small population size. The higher the number of marker loci assessed in a population genetic study, the better the accuracy of results $[31,32]$. Studies based on a few molecular marker loci have been reported to have a tendency to be biased [33]. In order to compensate for the dominant nature of AFLP markers, the presence and absence binary data were converted to expected heterozygosis through the assumption of Hardy-Weinberg equilibrium. This approach allowed us to obtain estimates that are almost as informative as those generated from co dominant markers. Furthermore, the criteria described by Lynch and Milligan were applied to the data to attain an unbiased estimate of heterozygosity.
Analyses of AFLP data revealed moderate levels of genetic variation among individuals in our study population. Identical patterns of genetic clustering of $M$. zahlbruckneri were obtained from both the UPGMA and NJ cladograms. Despite low bootstrap values at some of the UPGMA tree nodes, there was consistency in the pattern of clustering as shown by other methods used in this study with several individuals assigned to the same clusters with only minor overlap of samples between clusters. Model based Bayesian analysis provided additional information about the genetic structure of the population. Bayesian assignment and patterns of genetic variation suggested the presence of a moderately varied gene pool and the possibility of hybridization with closely related Melicope species based on the proportion of shared versus unique alleles across individuals in different cluster groups. In addition, the significant average FST values shown by two out of four clusters in the population further increased the likelihood of some external gene flow and the presence of individuals from other unidentified closely related Melicope species. The observed pattern was quite unexpected given the small population size and endangered status of the population. A plausible explanation for this observation is the high percentage of unique alleles detected in the eight individuals that showed the possibility of having mixed ancestry and a few others (particularly, MZ 23 and MZ 27) that may possibly belong to another species. It is noteworthy to mention that samples that showed the presence of unique alleles also had distinctly different morphological characteristics compared to the rest of the population. Additional genetic analysis is recommended to clarify the identity of these samples to determine their pedigree and species assignment.

The extent of overall genetic diversity detected in the population could also be attributed to historic gene flow that may have occurred with other closely related taxa when populations were more abundant and continuous, or it might be a result of more recent gene flow events with other Melicope species. It remains unknown how wide spread the observed unique alleles are with respect to other existing Melicope species. Geographic information showed that $M$. radiata is the closest population to M. zahlbruckneri in terms of physical distance. Although the present work did not examine $M$. radiata by molecular methods, there are strong indications based on morphological information that some level of gene flow may be occurring between these two species. It would be informative in future studies to obtain the nucleotide sequence of these unique alleles and assess their fixation in other populations.

The results presented here demonstrate that AFLP analyses in combination with appropriate statistics can detect genetic variability and provide genetic information that are valuable for conservation purposes in natural populations. Similar observations of relatively high to moderate genetic diversity in small populations have been reported in other tropical species e.g. Swietenia humilis [34] and Eucalyptus albens [35] and endangered populations [36,37]. A number of the authors attributed the observed level of genetic diversity to the outcrossing mating system of the species, which enabled pollination across isolated and small populations to influence gene flow. This is mostly characteristic of tree species with long distance seed and pollen dispersal [38]. Also, long term adaptation of small remnant populations to local conditions is a factor that can influence genetic diversity. There are clearly some limitations with using dominant molecular markers for genetic analysis compared with co-dominant markers; for example, their inability to provide locus information and distinguish between hybrids is apparent drawbacks [39]. Nonetheless, their ease of application across taxa in natural populations, ability to 
detect small genetic differences and genome-wide coverage has made them suitable and highly recommended as the markers of choice in circumstances where resources are limited to develop co dominant markers and where the study objective is to obtain initial information about overall genetic variation of a population. Moreover, their compatibility with the model based Bayesian approach for inferring genetic structure without prior information about the population has demonstrated that unbiased estimates of heterozygosity and genetic differentiation can be obtained.

Given the amount of variability detected in the M. zahlbruckneri population, it may be safe to reason that the genetic risk for this species is relatively moderate at present, but considering the small population size of the single remaining population, additional studies may be needed to examine the sustainability of the observed genetic diversity regarding longer term ecological impacts on the population distribution and habitat preservation. Furthermore, it is essential to understand that using only a few samples as founding individuals can considerably impact allelic diversity and result in higher genetic drift in the long run, therefore, representation of all identified $M$. zahlbruckneri individuals, including their putative hybrids in restorative planting at new sites would be beneficial to restoration success. Also, MZ23 and MZ27 may be excluded pending confirmation of their species identity.

Future studies to determine the level of available genetic variability across the species range would be desirable. This could be done by including other related taxa in the family Rutaceae for a comparative genetic analysis. Additionally, developing co-dominant marker systems such as Single Nucleotide Polymorphic (SNP) markers via the application of genotyping by sequencing technique as well as microsatellite markers may be useful for finer scale genetic studies of the endangered population to obtain phylogenetic information and comparative analysis of genomes between Melicope sp. and other closely related taxa. In addition, it would be beneficial to know genome sizes and ploidy levels of these species for a better understanding of the structure, organization and evolution of the species' genome. This information will better assist resource managers in planning conservation of this critically endangered species.

\section{Conclusion}

The results presented in this study illustrates the current genetic variation status of federally endangered $M$. zahlbruckneri and provides useful baseline information based on genetic and geographic population evaluation that can assist ongoing conservation and management priorities for the only known remaining population.

\section{Acknowledgement}

This work was supported in part by the U.S. Geological Survey Natural Resource Preservation (NRPP) and Wildlife and Terrestrial Resources Programs. The authors are grateful to Linda Pratt, Josh Van Demark, and Sierra McDaniel for assistance with field sample collections and for providing location information; and USGS Pacific Island Ecosystems Research Center for additional funds to support completion of the project. Any use of trade, product, or firm names in this publication is for descriptive purposes only and does not imply endorsement by the U.S. Government.

\section{References}

1. Bauert MR, Kalin M, Baltisberger M, Edwards PJ (1998) No genetic variation within isolated relict populations of Saxifraga cernua in the Alps using RAPD markers. Mol Ecol 7: 1519-1527.

2. Lynch M, Milligan BG (1994) Analysis of population genetic structure with RAPD markers. Mol Ecol 3: 91-99.

3. Reed DH, Frankham R (2003) Correlation between fitness and genetic diversity. Conserv Biol 17: 230-237.

4. Wagner WL, Herbst DR, Sohmer SH (1999) Manual of the flowering plants of Hawai'i. Revised edition. University of Hawai'i Press and Bishop Museum Press, Honolulu, HI, p: 1942.

5. Luan SS, Chiang TY, Gong X (2006) High genetic diversity vs. low genetic differentiation in Nouelia insignis (Asteraceae), a narrowly distributed and endemic species in China, revealed by ISSR fingerprinting. Ann Bot 98: 583-589.

6. Zawko G, Krauss SL, Dixon KW, Sivasithamparam K (2001) Conservation genetics of the rare and endangered Leucopogon obtectus (Ericaceae). Mol Ecol 10: 2389-2396.

7. Young A, Boyle T, Brown T (1996) The population genetic consequences of habitat fragmentation for plants. Trends Ecol Evol 11: 413-418.

8. Montgomery ME, Woodworth LM, Nurthen RK, Gilligan DM, Briscoe DA, et al.(2000) Relationships between population size and loss of genetic diversity: comparisons of experimental results with theoretical predictions. Conserv Genet 1: 33-43.

9. Cruzan MB (2001) Population size and fragmentation thresholds for the maintenance of genetic diversity in the endemic, Scutellaria montana (Lamiaceae). Evolution 55: 1569-1580.

10. Wang HW, Ge S (2006) Phytogeography of the endangered Cathaya argyrophylla (Pinaceae) inferred from sequence variation of mitochondrial and nuclear DNA. Mol Ecol 15: 4109-4122.

11. Raji A, Anderson JV, Kolade OA, Ugwu CD, Dixon AGO, et al. (2009) Gene-based microsatellites for cassava (Manihot esculenta Crantz): prevalence, polymorphisms, and cross-taxa utility. BMC Plant Biol 9: 118.

12. Yuan J, Cheng F, Zhou S (2012) Genetic structure of the tree peony (Paeonia rockii) and the Qinling Mountains as a geographic barrier driving the fragmentation of a large population. PloS ONE 7: e34955.

13. Robinson JP, Harris SA (1999) Amplified Fragment Length Polymorphisms and microsatellites: A phylogenetic perspective. Which DNA marker for which purpose? Chapter 12, Institute for Forest Genetics and Forest Plant Breeding, University of Gottingen, Gottingen, Germany, pp: $1-27$.

14. U.S. Fish and Wildlife Service (1997) Draft Big Island II: addendum to the recovery plan for the Big Island cluster. U.S. Fish and Wildlife Service, Portland, OR, p: 66.

15. Dellaporta SL, Wood J Hicks JB (1983) A plant DNA miniprepration: version II. Plant Mol Biol Rep 1: 19-21.

16. De Bang T, Raji A and Ingelbrecht I (2011) A multiplex microsatellite marker kit for diversity assessment of large Cassava (Manihot esculenta Crantz) germplasm Collections. Plant Mol Biol Rep 29: 655-662.

17. Varshney RK, Graner A, Sorrells ME (2005) Genic microsatellite markers in plants: features and applications. Trend Biotechnol 23: 48-55.

18. Katoh S, Kaneko S, Isagi Y, Murakami N, Kato H (2007) Isolation and characterization of microsatellite loci in Melicope quadrilocularis (Rutaceae), an endemic plant species of the Bonin Islands, Japan, and cross-species amplification in closely related taxa. Conserv Genet 8: 1487-1490.

19. Vos P, Hogers R, Bleeker M, Reijans M, van de Lee T, et al. (1995) AFLP: a new technique for DNA fingerprinting. Nucleic Acids Res 11: 4407-4414.

20. Sneath PHA, Sokal RR (1973) Numerical taxonomy. W. H. Freeman and Company, San Francisco, CA, USA.

21. Rohlf FJ (2005) NTSYS-pc: Numerical taxonomy and multivariate analysis system. Version 2.2k. Exeter Publications, New York, NY, USA.

22. Felsenstein J (2006) PHYLIP (Phylogeny Inference Package). University of Washington, Seattle, WA, USA. 
Citation: Raji JA, Atkinson CT (2016) Genetic Variation and Structure in Remnant Population of Critically Endangered Melicope zahlbruckneri. J Biodivers Endanger Species 4: 175. doi:10.4172/2332-2543.1000175

Page 8 of 8

23. Vekemans X (2002) AFLP-SURV. Version 1.0. Distributed by the author Laboratory of Genetics and Plant Ecology, University of Brussels, Belgium.

24. Pritchard JK, Stephens M, Donnelly P (2000) Inference of population structure using multilocus genotype data. Genetics 155: 945-959.

25. Falush D, Stephens M, Pritchard JK (2007) Inference of population structure using multilocus genotype data: dominant markers and null alleles. Mol Ecol Notes 7: 574-578.

26. Hubisz MJ, Falush D, Stephens M, Pritchard JK (2009) Inferring weak population structure with the assistance of sample group information. Mol Ecol Resour 9: 1322-1332.

27. Zhivotovsky LA (1999) Estimating population structure in diploids with multilocus dominant DNA markers. Mol Ecol 8: 907-913.

28. Dent AE, vonHoldt BM (2012) STRUCTURE HARVESTER: a website and program for visualizing STRUCTURE output and implementing the Evanno method. Conserv Genet Resour 4: 359-361.

29. Jakobsson M, Rosenberg NA (2007) CLUMPP: a cluster matching and permutation program for dealing with label switching and multimodality in analysis of population structure. Bioinformatics 23: 1801-1806.

30. Rosenberg NA (2004) Distruct: a program for the graphical display of population structure. Mol Ecol Notes 4: 137-138.

31. Xu S, Tauer CG, Nelson CD (2008) Genetic diversity within and among populations of shortleaf pine (Pinusechinata Mill) and loblolly pine (Pinustaeda L.). Tree Genet Genomes 4: 859-868.
32. Moser H, Lee M (1994) RFLP variation of genealogical dissimilarity, multivariate dissimilarity, heterosis and genetic variation in oats. Theor Appl Genet 87: 947-956.

33. Mariette S, Le Corre V, Austerlitz F, Kremer A (2002) Sampling within the genome for measuring within-population diversity: trade-offs between markers. Mol Ecol 11: 1145-1156.

34. White GM, Boshier DH, Powell W (1999) Genetic variation within a fragmented population of Swietenia humilis Zucc. Mol Ecol 8: 1899-1909.

35. Prober SM, Brown AHD (1994) Conservation of the grassy white box woodlands: population genetics and fragmentation of Eucalyptus albens. Conserv Biol 8: 1003-1013.

36. Breinholt JW, Buren VR, Kopp OR, Stephen CL (2007) Population genetic structure of an endangered Utah endemic, Astragalus ampullarioides (Fabaceae). Am J Bot 96: 661-667.

37. Duffy KJ, Fay MF, Smith RJ, Stout JC (2011) Population genetics and conservation of the small white orchid Pseudorchis albida in Ireland. Biology and Environment: Proceedings of the Royal Irish Academy 111B: 1-9.

38. Hamrick JL, Murawski DA, Nason JD (1993) The influence of seed dispersal mechanisms on the genetic structure of tropical tree populations. Adv Veg Sci pp: 281-297.

39. Dasmahapatra KK, Lacy RC, Amos W (2008) Estimating levels of inbreeding using AFLP markers. Heredity 100: 286-295. 